\title{
NO OPTICAL PULSES FROM THE GEMINGA CANDIDATE ${ }^{a}$
}

\author{
Shrinivas R. KUlKarni AND S. DJORgovski \\ Department of Astronomy and Radio Astronomy Laboratory, University of California, Berkeley, California 94720 \\ Received: 26 July 1985; revised 6 September 1985
}

\begin{abstract}
We have conducted several optical studies of a 21 mag star suggested to be the optical counterpart of the pulsed x-ray source 1 E $0630+178$ and the $\gamma$-ray source 2CG $195+04$ (Geminga). We report the absence of significant optical pulsed emission over the entire period range claimed from the analysis of high-energy data. We also find no significant variability on time scales from tens of minutes to a few months. We suggest that the true optical counterpart of the $\mathrm{x}$-ray source is either one of the two fainter stars in the $x$-ray source error box or an as yet undetected fainter star. In either case, $1 \mathrm{E} 0630+178$ is a unique $x$-ray source because its ratio of $L_{x}(0.2-2.0 \mathrm{keV}) / L_{v}(3000-7000 \AA)$ is at least 1100 , which is characteristic of galactic-bulge x-ray sources. However, its absolute magnitude is at least about $M_{v} \sim 20$ and not $M_{v} \sim 3 \mathrm{mag}$, typical of the bulge sources.
\end{abstract}

\section{INTRODUCTION}

The $\gamma$-ray source $2 \mathrm{CG} 195+04$, first discovered by the $S A S-2$ satellite and confirmed by the $C O S-B$ satellite, is the brightest unidentified source in the $C O S-B$ catalog (Bignami and Hermsen 1983). Followup of the $C O S-B$ error circle ( 0.4 radius) by the Einstein $\mathrm{x}$-ray satellite led to the discovery of four sources (Bignami, Caraveo, and Lamb 1983). Of these sources, $1 \mathrm{E} 0630+178$, the brightest, was considered to be the most probable $x$-ray counterpart to Geminga based on (a) an unusually soft $x$-ray spectrum (restricting the distance to less than $100 \mathrm{pc}$ ) and (b) on a low probability of a pure chance appearance of such a high-flux-density source within the COS-B error circle (Bignami, Caraveo, and Lamb 1983). Optical investigation of the Einstein HRI (High-Resolution Imager) error circle revealed a $m_{v} \sim 21$ mag stellar object just outside the $90 \%$ probability error circle and this star has been proposed as the optical candidate for $1 \mathrm{E}$ $0630+178$ (Caraveo et al. 1984). Following this identification, this object has been pursued vigorously by several groups (Bloemen 1984; Vigroux et al. 1984; Sol et al. 1985; Halpern, Grindlay, and Tytler 1985). We will henceforth refer to this source as the "G candidate" after the convention of Vigroux et al. (1984) and Sol et al. (1985).

There has been considerable controversy about the temporal nature of the signal from Geminga at $\gamma$-ray wavelengths and at $\mathrm{x}$-ray wavelengths from the reputed $\mathrm{x}$-ray candidate. A period of $\approx 59 \mathrm{~s}$ was originally reported by the SAS-2 team (Thompson et al. 1977), was later confirmed (Masnou et al. 1977), and then retracted by the COS-B collaboration (Masnou et al. 1981). Recently the periodicity issue has resurfaced with an announcement by Bignami, Caraveo, and Paul (1984, hereafter referred to as BCP) of a discovery of deep $(\sim 40 \%)$, pulsed emission from an analysis of the Einstein and the Exosat satellite data of $1 \mathrm{E} 0630+178$. However, Bucheri et al. (1985) dispute the statistical significance of the findings of BCP.

Accepting the results of $\mathrm{BCP}$, we have conducted several observations of the $x$-ray error box at the Lick $3 \mathrm{~m}$ Shane telescope. In this paper we report the results of a search for periodic optical emission from the $\mathrm{G}$ candidate; other results are reported in the companion paper (Djorgovski and Kulkarni 1986, hereafter referred to as DK).

a) Based in part on research done at Lick Observatory, University of California.

98

Astron. J. 91 (1), January 1986

$0004-6256 / 86 / 010098-09 \$ 00.90$

The conventional technique of using single-channel photometers for a periodicity search becomes less attractive when the object is comparable to, or fainter than, the night sky (typically 20-21 mag per $\operatorname{arcsec}^{2}$ ) for several reasons: (a) low quantum efficiency (typically 10\%), (b) for any reasonable aperture (5-10 arcsec) the contribution from the sky is overwhelming, and (c) accurate estimation of the dominant, time-variable sky contribution is generally not possible. In comparison, two-dimensional detectors such as charge-coupled devices (CCD) do not suffer from these problems since systematic effects that arise from an inhomogeneous sky background and variable seeing and transparency can be minimized by doing local sky subtraction and relative photometry with respect to the other stars in the field of an optical candidate; additionally, optical apertures such as one weighted by the point-spread function can be employed-leading to further improvements in the signal-tonoise ratio. Unfortunately, CCD cameras cannot be read out frequently without a loss in observing time and without an increase in the effective noise caused by frequent readouts. We circumvented this difficulty with a somewhat novel scheme, which is applicable only when a rough period is $a$ priori known. The assumed putative period $\left(P_{0}\right)$ is broken into a certain number of bins (say $m$ bins), each with a width of $P_{0} / \mathrm{m}$. In order to measure the light curve in one of these phase bins (say the $i$ th bin), the shutter of the CCD camera is opened, once every putative period, for an interval that corresponds to the phase bin in question; for the rest of the putative period the shutter is closed (see Fig. 1). This sequence is continued for $n$ periods and then the CCD image is read out and recorded. This procedure is repeated for $i=1,2, \ldots, m$ to yield the entire light curve. Note that the duty cycle of such observations is $1 / \mathrm{m}$.

We now discuss the choice of the parameters and the details of the "shutter controller" employed for the G-candidate observations. A programmable synthesizer (ADRET 3310) whose frequency was set to $2^{23} P_{0}^{-1} \mathrm{~Hz}$ was used for generation of control signals for the shutter; here $P_{0}$ is the putative period in seconds. The sine-wave output of the synthesizer was shaped to a square wave, converted to the usual TTL (transistor-transistor logic) levels (i.e., 0 and $+5 \mathrm{~V}$ ) and then fed to a 24-bit digital, binary counter. The outputs of the two most significant bits of the binary counter corresponding to $50 \%$ duty cycle, rectangular waveforms were 


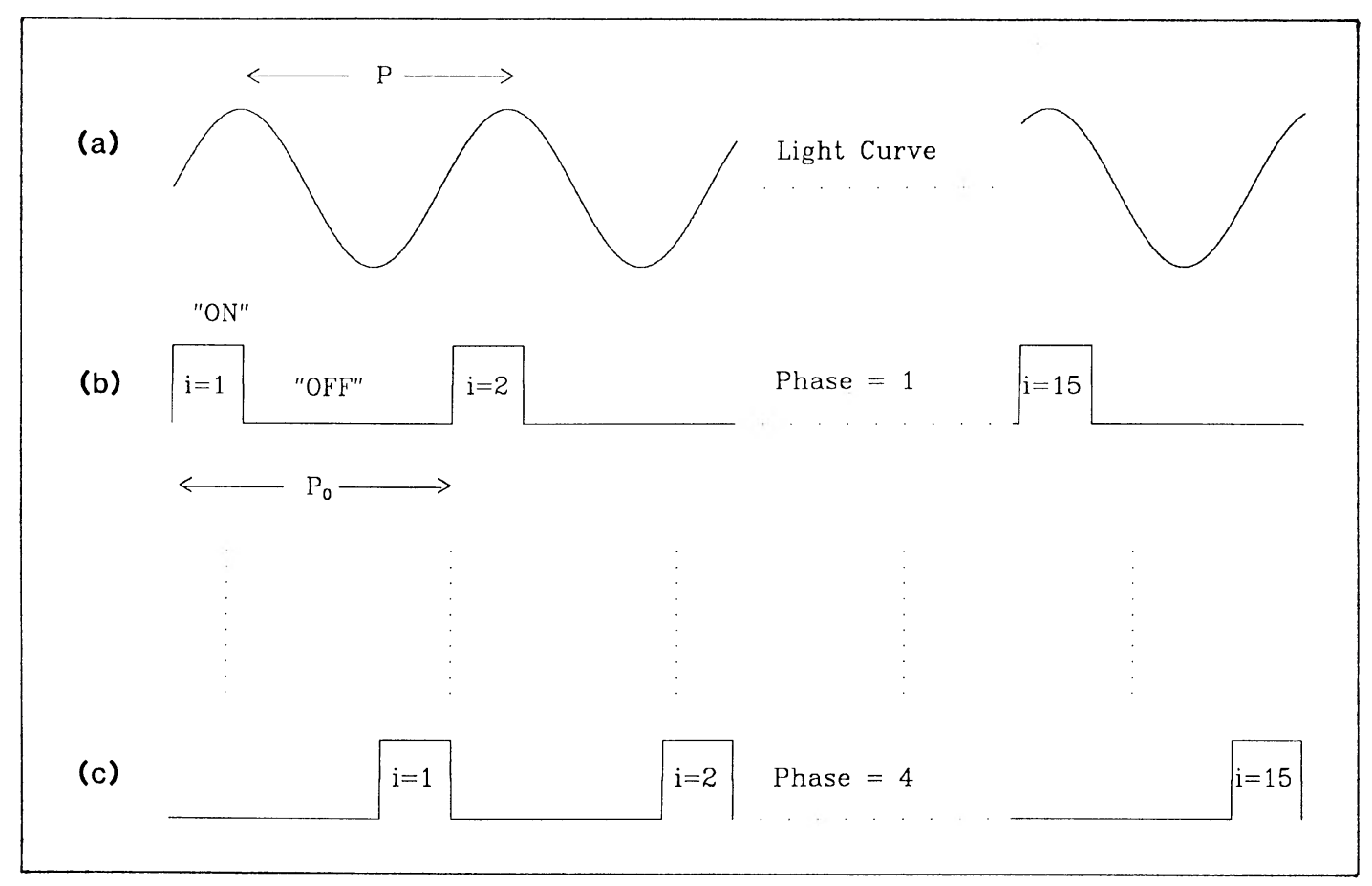

FIG. 1. Schematic illustration of the CCD data-acquisition system used to measure the light curve of the $\mathrm{G}$ candidate, assumed to have a period $P$ [Fig. 1(a) ]. This system can measure the light curve averaged over four phase bins each one-quarter $P_{0}$ wide. Here $P_{0}$ is an approximate period that is a priori known. In order to measure the light curve over the first quarter $P_{0}$, the shutter is closed and opened in the sequence shown in Fig. 1 (b). Figure 1 (c) illustrates the case for phase bin $=4$. For the G-candidate observations, after 15 such "onoff' cycles, the CCD frame was read out. Differences between $P$ and $P_{0}$ lead to phase differences and this can be clearly seen towards the end of the integration ( $i=15$ ); here $P_{0}$ was chosen to be $5 \%$ smaller than $P$.

used to address a 1:4 demultiplexer whose input was connected to $+5 \mathrm{~V}$. The resulting signals from two of the four outputs are shown in Figs. 1(b) and 1(c). A 4:1 rotary switch selected one of these outputs. A master toggle switch then allowed us to choose between this resulting output and $0 \mathrm{~V}$-the "shutter-close" position. The output of the shutter controller was fed to the electromechanical shutter of the CCD camera; it was also connected to a light-emitting diode (LED) to serve as a visual indicator of the state of the shutter.

We chose $m=4$ as a compromise between higher resolution of the light curve and a desire to have as large a duty cycle as possible. The resulting resolution was sufficiently fine enough to measure an optical light if it was similar to the $\mathrm{x}$-ray light curve (see Fig. 2 in BCL). The synthesizer frequency was set to $139194.3 \mathrm{~Hz}$ corresponding to $P_{0}=60.265456 \mathrm{~s}$. For each of these phase bins we integrated for a total time of $15 \times P_{0}$. Reasons for the choice of $P_{0}$ and $n$ are discussed below.

The data for this study were obtained on the nights of 23 and 24 February 1985 (UT) with the Cassegrain CCD on the $3 \mathrm{~m}$ Shane telescope of the Lick Observatory (Miller 1983); the journal of the observations is given in Table I. All the imaging observations were done using a wide (equivalent

TABLE I. Journal of observations.

\begin{tabular}{|c|c|c|c|c|}
\hline $\begin{array}{c}\text { Observation } \\
\text { No. }\end{array}$ & UT date & Data type & Exposures & $\begin{array}{l}\text { Seeing } \\
\text { (arcsec) }\end{array}$ \\
\hline $\begin{array}{l}1 \\
2 \\
3 \\
4 \\
5 \\
6 \\
7\end{array}$ & $\begin{array}{l}26 \text { November } 1984 \\
21 \text { December } 1984 \\
28 \text { December } 1984 \\
29 \text { December } 1984 \\
23 \text { February } 1985 \\
24 \text { February } 1985 \\
25 \text { February } 1985\end{array}$ & $\begin{array}{l}\text { Image } \\
\text { Image } \\
\text { Image } \\
\text { Spectrum } \\
\text { Image } \\
\text { Spectrum } \\
\text { Image } \\
\text { Image (phased) } \\
\text { Image } \\
\text { Image (phased) } \\
\text { Image }(\mathbf{H} \alpha)\end{array}$ & $\begin{array}{l}4 \times 300 \mathrm{~s} \\
2 \times 300 \mathrm{~s} \\
3 \times 300 \mathrm{~s} \\
4000 \mathrm{~s} \\
4 \times 300 \mathrm{~s} \\
1300 \mathrm{~s}, 4000 \mathrm{~s} \\
300 \mathrm{~s} \\
8 \times 904 \mathrm{~s} \\
2 \times 300 \mathrm{~s} \\
8 \times 904 \mathrm{~s} \\
2 \times 1200 \mathrm{~s}\end{array}$ & $\begin{array}{r}1.5 \\
1.4 \\
1.8 \\
1.4 \\
1.5 \\
1.2 \\
\sim 1.2\end{array}$ \\
\hline
\end{tabular}

Notes to TABLE I

All data reported here were obtained with the Cassegrain CCD camera of the Lick Observatory. The images were obtained with the wide, red filter described in the text. The $\mathrm{H} \alpha$ images were obtained with a $100 \mathrm{~A}$-wide filter centered on the rest $\mathrm{H} \alpha$ wavelength. The "phased" images referred to the images obtained with the shutter controller described in the text. Column 4 is the seeing radius and this is defined to be the radius at which the surface brightness falls to half the central surface brightness. Owing to the large pixel size $(0.73$ arcsec), the central surface brightness is underestimated; this results in an overestimation of the seeing radius. 
width $=1370 \AA$ ), red filter with $\lambda_{\text {eff }}=6890 \AA$; see Djorgovski (1985) for additional details about this filter. We started the observations with first-phase-bin observations and proceeded sequentially to the fourth phase bin; this cycle was then repeated once more. This resulted in a total of 16 CCD images-eight images each night. For each cycle of four images, the camera position was held steady, so that each star was exposed to the same set of pixels. For each exposure we followed exactly the same procedure: about $10 \mathrm{~s}$ before we anticipated the shutter-controller output to go high and hence be capable of opening the shutter, the master switch was toggled to the shutter-controller position; the exact moment ( to $\pm 1 \mathrm{~s}$ ) when the LED lit up was then noted (visually) in our log book. The shutter-controller box was reset sometime in the evening of 23 February 1985, and after that was left running continuously. For each observation, the high stability of the frequency synthesizer allowed us to deduce the exact time (to $\pm 1 \mathrm{~ms}$ ) at which the shutter opened for the first time. After the shutter had completed 15 “on-off" cycles we toggled the master switch to the shutterclose position and read out the CCD. As a check on the accuracy of the synthesizer, $P_{0}$ was independently estimated by noting down the time interval between a great number $(\sim 3000)$ of integer cycles of the shutter output. The good agreement between this estimate of $P_{0}$, which is tied to the WWV (a radio time standard in the U.S.A.) clock, and the one deduced from the frequency setting of the synthesizer meant that there was no drift, of purely instrumental origin, between our synthesizer-based clock and the "Geminga clock."

\section{DATA REDUCTION}

The 16 CCD images were subjected to the standard datareduction procedure of cosmic-ray removal and division by a flat field. In Fig. 2 we present a stack of some of the bestseeing CCD images. Photometry was performed for ten stars in each field-six bright stars (A through F), the $G$ candidate and three stars as faint as the $G$ candidate (Comp. 1, Comp. 2, and Comp. 3; see Fig. 2). In Table II, we present the positions and the magnitudes of all the secondary stars in the field. For this particular table, we carefully chose a CCD image in which the six bright stars had not bled. On the particular frame we had chosen, due to the artifact of the CCD detector we could not reliably measure the magnitude or position of Comp. 3. Nor could we measure the magnitude of star 6, given its proximity to the extremely bright star 4. However, the image of star Comp. 3 did not suffer any such problem in the 16 "phased" images obtained for the purpose of studying temporal variations of the $\mathrm{G}$ candidate.

For every single one of the 16 phased frames, the run of surface brightness versus aperture radius for the fainter stars were checked and an aperture size chosen (between 3 and 4 arcsec) as a compromise between sky and stellar contributions; the instrumental magnitudes were then measured in that aperture. The flux standards Feige 56 and EG 247 of Stone (1977) were used to convert these instrumental magnitudes to the $r_{s}$ magnitudes (see Djorgovski 1985 for a definition of the $r_{s}$ system). The positions of the stars listed in Table II were derived from a grid of 31 nearby SAO stars.

For the search for optical modulation, the instrumental magnitudes of the three comparison stars and the $\mathbf{G}$ candidate were defined relative to the five bright stars. This artifice renders the relative magnitudes impervious to variations in sky transparency and brightness, drifts in detector perfor- mance, etc. As an additional precaution, we obtained these 16 images within $\pm 1 \mathrm{hr}$ of the transit of the $\mathrm{G}$ candidate. Thus we believe that the relative magnitudes should be limited by photon statistics. In Fig. 3, we show the relative magnitudes of the comparison stars and the $\mathrm{G}$ candidate. The plotted instrumental magnitudes have an arbitrary constant subtracted for each star so that the mean relative magnitude for each is exactly zero.

\section{RESULTS}

A preliminary analysis of the results was reported earlier (Kulkarni and Djorgovski 1985). Here, we analyze, in detail, the data shown in Fig. 3. A first glance at this figure does not reveal any outstanding light curve for the $\mathbf{G}$ candidate. In fact, the rms in the relative magnitude $\sigma_{\Delta m}=0.07 \mathrm{mag}$ for the $\mathrm{G}$ candidate is no different from that of the comparison stars, which are of comparable brightness. In contrast, the $\mathrm{x}$-ray light curve of $1 \mathrm{E} 0630+178$ has a peak-to-trough variation of $2.5: 1$ or nearly a magnitude. Thus, the first result is that the $\mathrm{G}$ candidate does not have a light curve of the same strength as the $\mathrm{x}$-ray source, at a period close to $P_{0}$. The analysis of high-energy data (BCP) indicates not only a $P$ but also a $\dot{P} \sim+4 \times 10^{-9} \mathrm{~s}^{-1}$. Since our technique is sensitive only to a restricted range around the assumed period, it was important for us to use the best possible guess for $P_{0}$. We did a simple least-squares fit to the periodicity data given by BCP and estimated $P=60.265$ s for the epoch of our optical observations. We now estimate the response of our observations to periods other than $60.265 \mathrm{~s}$ by modeling the entire observing procedure extensively.

For the purpose of modeling, we assume that the light curve can be represented by a sinusoidal function with an amplitude of $1 \mathrm{mag}$. We then estimate the amplitude that would have been measured by our observing system. The primary attenuation of response to periods other than $P_{0}$ arises from the shutter sequence of the "on-offs"; the bandpass introduced by this "filter" is illustrated in Fig. 4(a). The competition between the bandpass width (inversely related to $n$ ) and the need to detect the $G$ candidate with a reasonable signal-to-noise ratio (proportional to $\sqrt{n}$ ) led to a compromise choice of $n=15$. The irregular timing of the readout of the CCD frames introduces aliasing and irregular response to periods other than $P_{0}$. Analysis of irregular sampled data is also sensitive to the shape and the relative phase of the light curve with respect to our clock. The former problem appears not to be well appreciated in the literature. In the absence of the knowledge of the absolute $x$-ray phase of $1 \mathrm{E} 0630+178$, we have estimated the expected response by assuming the phase to be successively $0^{\circ}, 90^{\circ}, 135^{\circ}, 180^{\circ}$ at the time when our shutter controller was reset, viz. at our fiducial time. Given our value of $m$, finer subdivision of phase is unnecessary. Note that negative initial-phase values provide no additional information in the estimation of the amplitude-a positive-definite quantity. The $x$-ray light curve can be reasonably approximated by a sinusoid with a constant offset (see Fig. 2 of BCP). Thus if the optical light curve is assumed to be similar to the $x$-ray light curve, then our modeling is accurate from all aspects. Note that our data shown in Fig. 3 are insensitive to any offset in the light curve.

Assuming a period, we estimated the expected amplitude of the model light curve at each of the 16 observed times; from these amplitudes an rms, $\sigma_{\text {mod }}$, was then estimated; this procedure was then repeated for the three other initial phases. The response of our data-acquisition system, as mea- 


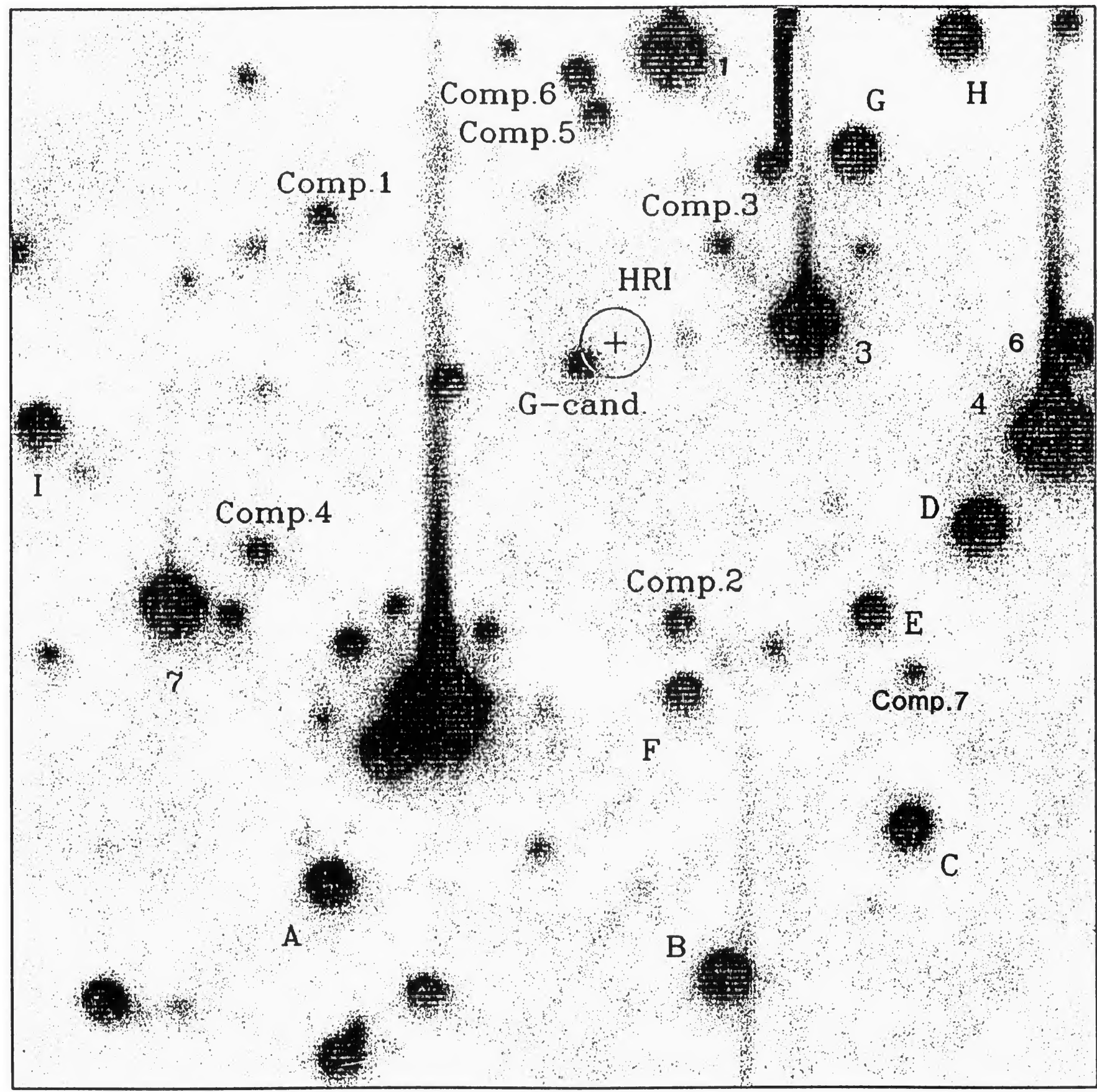

FIG. 2. CCD image of the G-candidate field. North is up and east is to the left; the field is a square of 103 arcsec on a side. The cross represents the Einstein HRI position of the X-ray source 1E $0630+178$ and the circle is the HRI $90 \%$ error circle. The star marked as "G-cand." has been considered to be a possible optical candidate for Geminga. The magnitudes and the positions of the marked stars used for relative photometry are given in Table II.

sured by the rms, to a range of periods about $P_{0}$ is shown in Fig. 4(b). This plot illustrates that our observations are sensitive to periods from 59.17 to $61.35 \mathrm{~s}$. Outside this range, $\sigma_{\text {mod }}$ ranges from $\sim 1$ (perfect response) to $\sim 0$ (no response); the shape of this response curve mainly arises from the effects of irregular sampling and this response curve is applicable regardless of the detailed shape of the light curve. For example, the crests and the troughs seen in Fig. 4(b) have a separation of about $12 \mu \mathrm{Hz}$ and represent the modulation caused by the observations separated by one full day (1/ $86400 \mathrm{~s}=12 \mu \mathrm{Hz}$ ).

The rms is not necessarily the best estimator for a purely sinusoidal wave. However, it has the virtue of being somewhat model independent. Since the rms for the $G$ candidate is not, within errors, different from that of the comparison stars, we can place a severe restriction on the rms of the light curve for an arbitrary shape. For Gaussian noise, the uncertainty on the rms is rms $/ \sqrt{p}$, where $p$ is the number of observations. Thus a $3 \sigma$ upper limit to the rms of an arbitrary light curve is $3 \times 0.07 \mathrm{mag} / \sqrt{16}=0.05 \mathrm{mag}$ or $5 \%$.

We now estimate the maximum possible amplitude allowed by our observations for the $\mathrm{G}$ candidate, assuming a sinusoidal light curve which, as stated before, is a fair approximation to the $x$-ray curve. Using the same modeling procedure as before, we estimated the amplitude $(A)$ and its uncertainty $\left(\sigma_{A}\right)$ by doing a least-squares fit between the model amplitudes and the observed relative magnitude for 
TABLE II. Photometry of the $\mathbf{G}$ candidate field.

\begin{tabular}{|c|c|c|c|}
\hline Star Id. & R.A. (1950) & Dec. (1950) & $r_{s}$ \\
\hline $\begin{array}{l}\text { Star A } \\
\text { Star B } \\
\text { Star C } \\
\text { Star D } \\
\text { Star E } \\
\text { Star F } \\
\text { Star G } \\
\text { Star H } \\
\text { Star I } \\
\text { Star 1 } \\
\text { Star 3 } \\
\text { Star 6 } \\
\text { Star 7 } \\
\text { Comparison 1 } \\
\text { Comparison 2 } \\
\text { Comparison 4 } \\
\text { Comparison 7 } \\
\text { G candidate } \\
\text { G' candidate }\end{array}$ & $\begin{array}{l}63101.03 \\
63058.43 \\
63057.12 \\
63056.71 \\
63057.46 \\
63058.70 \\
63057.56 \\
63056.83 \\
63102.98 \\
63058.76 \\
63057.88 \\
63056.12 \\
63102.10 \\
63101.09 \\
63058.73 \\
63101.52 \\
63057.17 \\
63059.38 \\
63059.02\end{array}$ & $\begin{array}{l}174741.79 \\
174732.76 \\
174747.12 \\
174816.39 \\
174807.19 \\
174759.72 \\
174850.78 \\
174901.06 \\
174825.88 \\
174900.27 \\
174834.76 \\
174833.11 \\
174808.63 \\
174844.87 \\
174806.31 \\
174813.41 \\
174801.23 \\
174830.84 \\
174832.39\end{array}$ & $\begin{array}{l}18.76 \\
18.04 \\
18.87 \\
17.73 \\
19.75 \\
19.81 \\
18.11 \\
18.12 \\
18.74 \\
16.64 \\
16.61 \\
* \\
17.23 \\
20.77 \\
21.0 \\
21.1 \\
22.4 \\
20.5 \\
24.5\end{array}$ \\
\hline
\end{tabular}

Notes to TABLE II

The uncertainty in the positions is estimated to be \pm 0.4 arcsec in each coordinate. In col. 4 , the star marked with an asterisk does not have photometry measurements. The uncertainty in the magnitude for stars brighter than 19 mag is about 0.02 mag; the uncertainty for the $G$ candidate and the four comparison stars is 0.07 mag. The magnitude of the $\mathrm{G}^{\prime}$ candidate is uncertain by about 0.50 mag.

the $\mathrm{G}$ candidate. Thus for each assumed period, and for each of the four choices of the initial phases, we get four amplitudes and four uncertainties on the amplitudes. In Fig. 5 we plot $A_{\max }, A_{\max }+3 \sigma_{A, \max }$ and $A_{\max }+3 \sigma_{A, \min }$; here $A_{\max }$ is the maximum of the four amplitudes, and likewise $\sigma_{A, \max }$ and $\sigma_{A, \min }$ are the maximum and minimum of the four uncertainties. From this plot, we conclude that in the worst possible case (i.e., $A_{\max }+3 \sigma_{A, \max }$ ), the maximum allowed amplitude of the $\mathrm{G}$ candidate is about $0.13 \mathrm{mag}$ or $13 \%$; or more fairly, the $3 \sigma$ limit on $A_{\max }$ is about $10 \%$ for $1 / P$ between 16.4 and $16.8 \mathrm{mHz}$. This limit could perhaps be improved by the use of point-spread-function weighted aper- tures in photometry. Anyway, the key result is that the $G$ candidate does not have the same light curve as the $\mathrm{x}$-ray light curve over the entire range of periods claimed by analysis of the very first $\gamma$-ray data (Thompson et al. 1977) to the latest $x$-ray data $(B C L)$ and the expected period at the current epoch.

As a by-product of our observations we were able to monitor the flux from the $\mathrm{G}$ candidate on longer time scales of tens of minutes to months (see Table I and Fig. 6). As can be seen from Fig. 6, the $G$ candidate does not show any outstanding variability in comparison to the equally faint comparison stars. Thus over time scales ranging from 1 day to 3

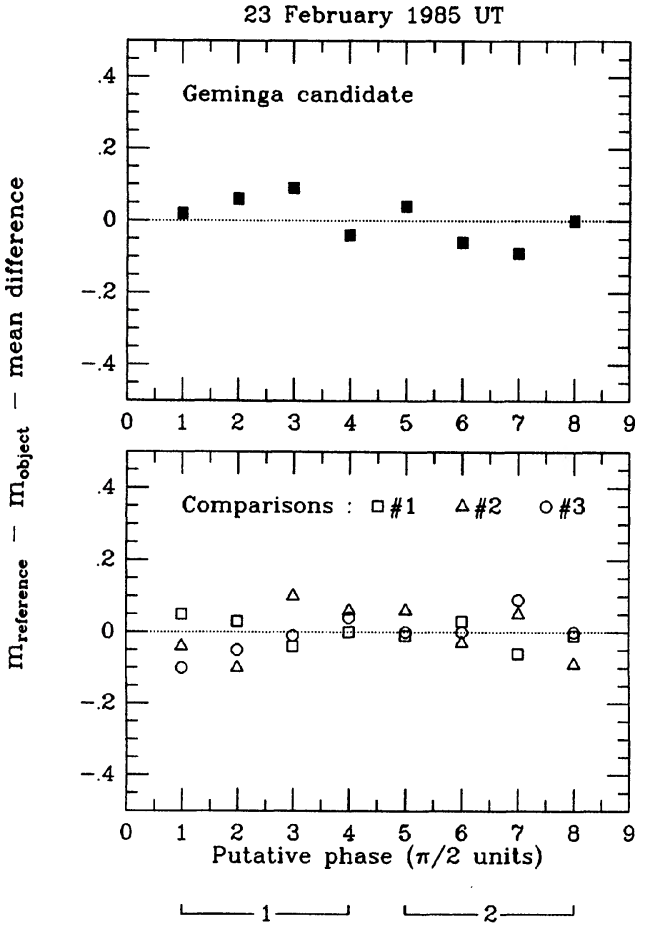

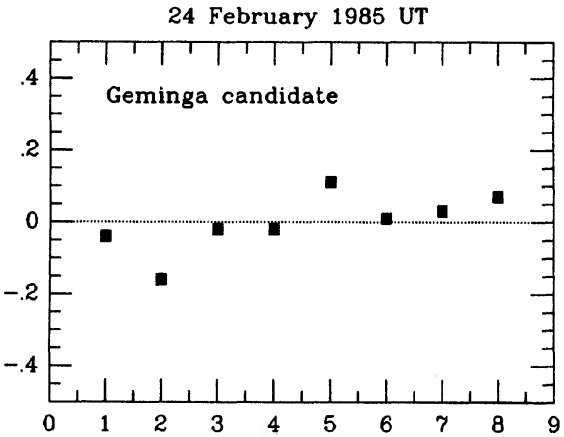

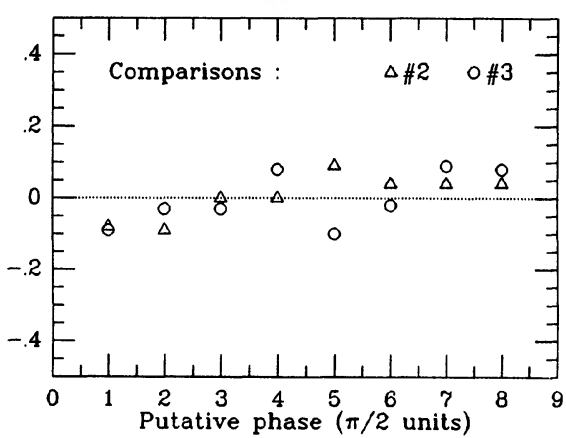

$\longleftarrow 1-2-$
Fig. 3. Relative magnitudes of the $\mathrm{G}$ candidate and the comparison stars as a function of the phase of the putative period for the two nights of observations. The plotted relative magnitudes are defined with respect to the bright stars $A$ through F; additionally, for each star, the relative magnitudes are forced to have a zero mean. 

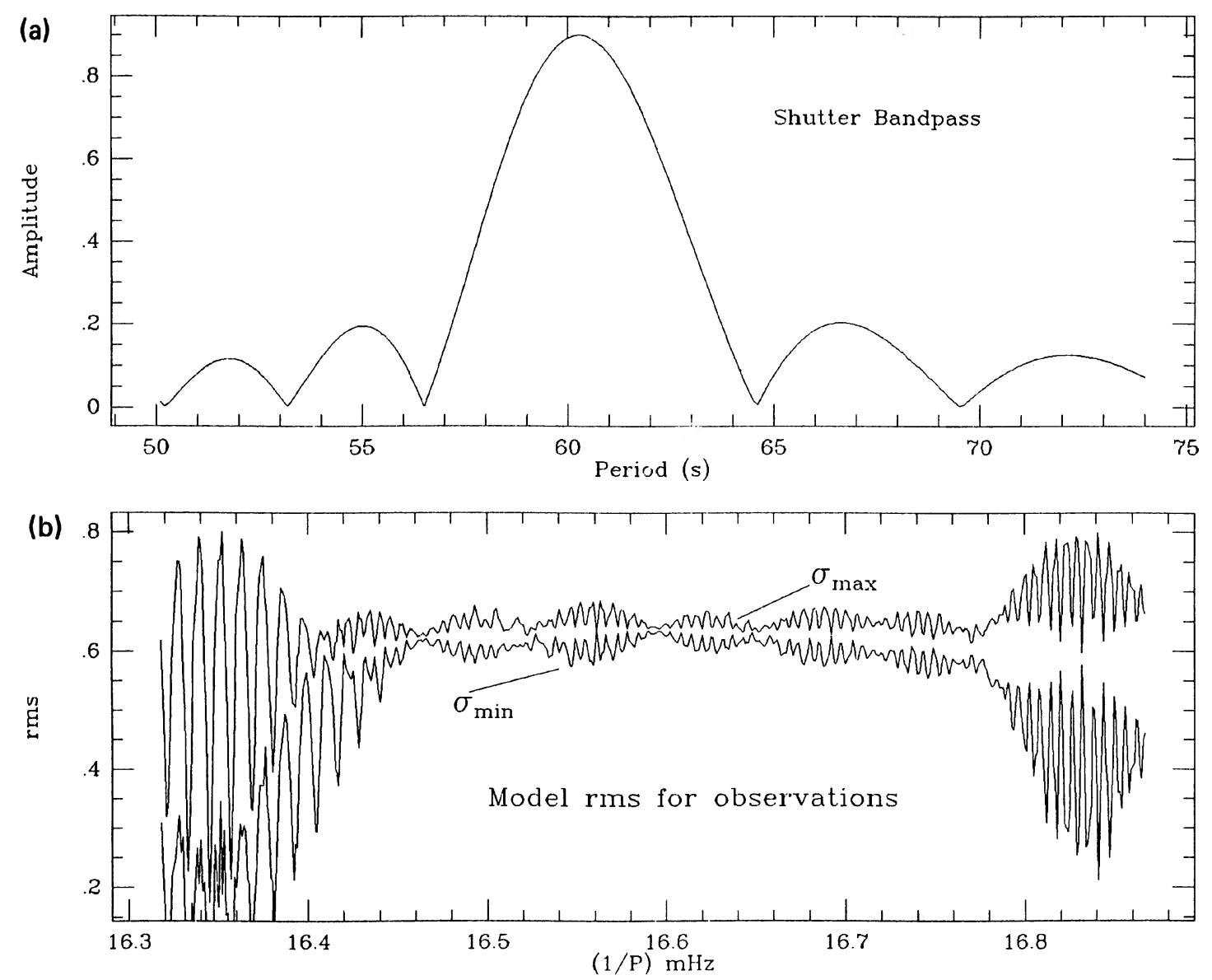

Fig. 4.(a) Response to periods other than $P_{0}$ arising from the sequence of 15 "on-off."(b) The final response incorporating the shutter sequence, and that arising from a sparse sampling of the CCD frames ( 16 frames over two nights). The light curve is assumed to be a sinusoid of unit amplitude and the expected amplitudes are calculated; the response is measured by the rms of these amplitudes. The response is sensitive to the initial phase of the light curve and this is illustrated by showing the worst $\left(\sigma_{\min }\right)$ and the best $\left(\sigma_{\max }\right)$ response. Thus our observations were sensitive to periods ranging from 59.17 to $61.35 \mathrm{~s}$.

months we do not find no more than $10 \%(1 \sigma)$ variation in the $\mathrm{G}$ candidate. This result is in agreement with that of Sol et al. (1985) and is not inconsistent with that of Vigroux et al. (1984).

\section{DISCUSSION AND CONCLUSIONS}

At visual wavelengths we have looked for periodic light emission from the $\mathrm{G}$ candidate-a proposed optical candidate (Caraveo et al. 1984). After a thorough analysis of our data, we severely limit the extent of any periodic emission from this source: if the optical light curve is similar to the Einstein x-ray curve, then the optical amplitude is $2 \% \pm 3 \%$ for periods between 59.9 and $60.6 \mathrm{~s}$; over a wider range in period from 59.5 to $61.0 \mathrm{~s}$, our limits are $4 \% \pm 3 \%$. The limit on the mean amplitude is biased towards higher values and hence these limits are more stringent than indicated. These results lead us to conclude that (a) the $x$-ray and the $\gamma$-ray periods are spurious and the $G$ candidate may still be the true optical counterpart or (b) the optical light curve is significantly shallower than the $x$-ray curve and that the $G$ candidate is indeed 1E $0630+178$, and finally (c) the true optical counterpart is some other star lurking in the HRI error circle.
The first possibility is not easy to rule out. Indeed the periodicity issue is filled with claims and counterclaims. In fact, recently Bucheri et al. (1985) reported that the periodicity analysis of all the high-energy ( $x$-ray and $\gamma$-ray) data by BCP do not indicate either a statistically significant period, let alone $\dot{P}$. Thus while we cannot offer any additional data to prove or disprove the analysis of BCP, we can and will discuss below possibilities (b) and (c).

In order to explain the claims of periodic $\mathrm{x}$-ray emission, several binary models have been proposed: a magnetic and a nonmagnetic neutron-star system (Nulsen and Fabian 1984), a white dwarf and a black hole system (Bisnovaty-Kogan 1985), and finally, a magnetic white dwarf and a nonmagnetic white dwarf (Arons 1985). In these models, $P$ and $\dot{P}$ arise from a combination of the binary orbital motions and/or the spin motions. Thus, if the geometry of the system is mainly responsible for the shape of the light curve, we expect the light curve to be relatively independent of the wavelength and in this context our null result acquires significance. A single neutron-star model has also been advanced for $\mathrm{Ge}$ minga (Maraschi and Treves 1977). In such models, the $x-$ ray curve may arise from a rotation of the neutron star; note that the optical radiation is more than $50 \%$ pulsed for the 


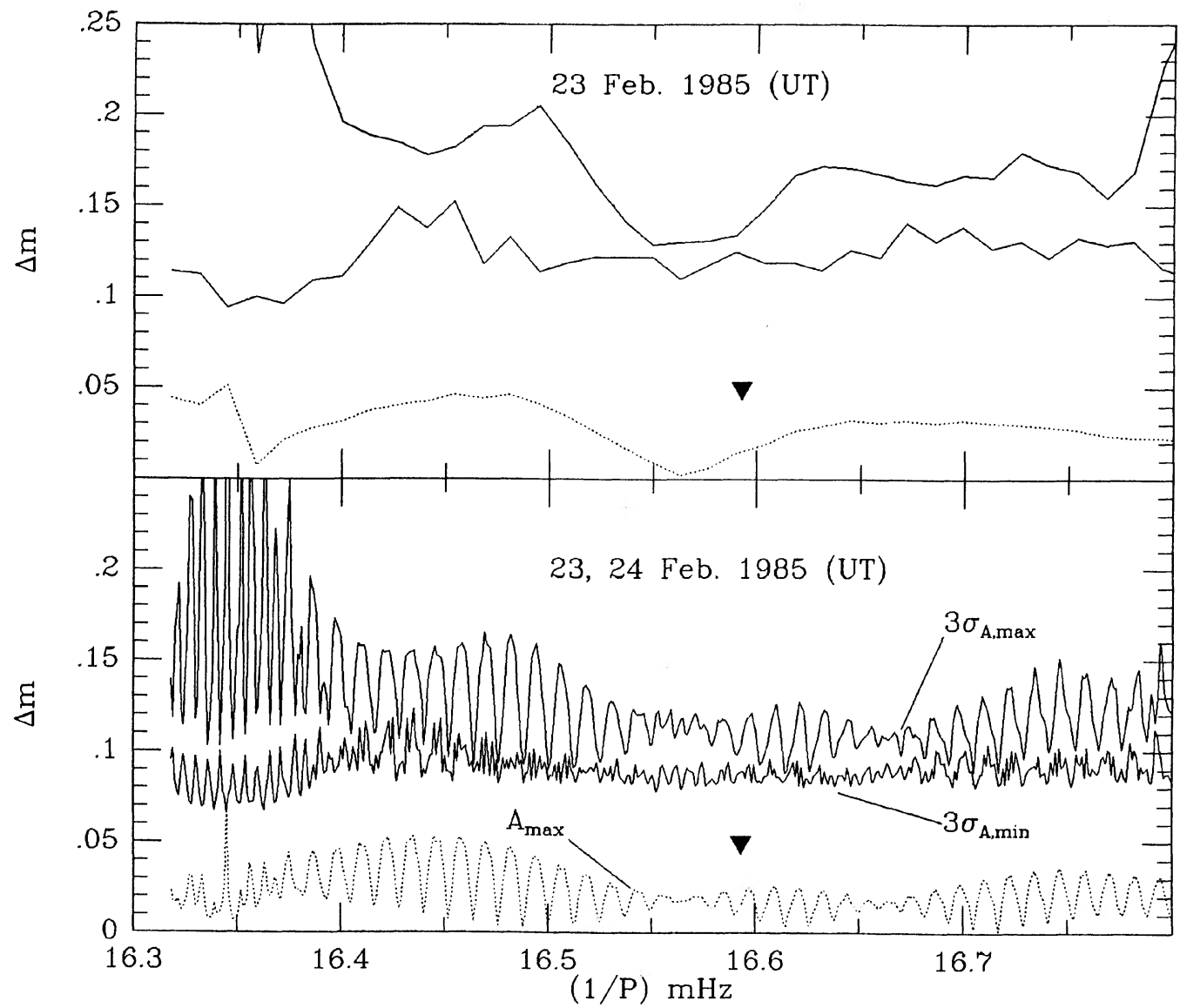

FIG. 5. Assuming that the optical light curve for Geminga could be approximated by a sinusoid, an amplitude $(A)$ and its rms $\left(\sigma_{A}\right)$ can be obtained for various assumed periods and assumed initial phases. In both the figures, the topmost solid line is a run of $A_{\max }+3 \sigma_{A, \max }$, the middle solid line is the quantity $A_{\max }+3 \sigma_{A, \min }$, and the dotted line is simply $A_{\max }$; these quantities are defined in the text. The dark triangle marks the period of $1 \mathrm{E} 0630+178$ expected at the epoch of our observations.

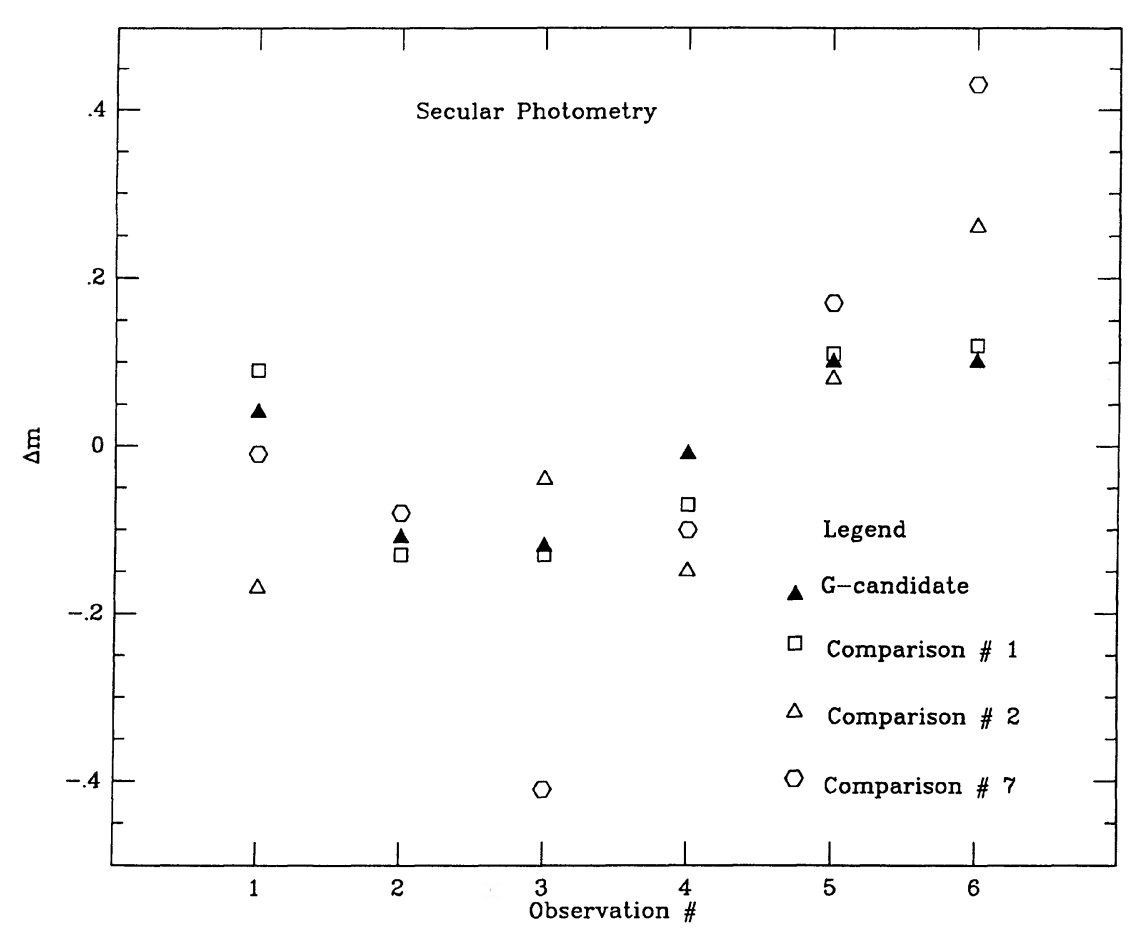

FiG. 6. Secular photometry of the G candidate and three comparison stars. The ordinate refers to the magnitudes of the stars relative to the bright stars A through F. For each of the four stars, the zero point is defined so as to yield a zero mean. 
two known, galactic, optical pulsars (Peterson et al. 1977). However, the large loss of rotational energy from such a system has led to the suggestion that precession is responsible for the light curve (Maraschi and Treves 1977). Again in this case, the orientation of the magnetic axis and the rotation axis, i.e., the geometry, dominates the shape of the light curve. Thus we conclude that in the context of either a single or double neutron-star model, our observations make point (a) unlikely.

Finally the optical radiation could be arising from a region different from the $\mathrm{x}$-ray and the $\gamma$-ray-emitting regions. We cannot rule out this possibility. However, it should be kept in mind that radiation from $1 \mathrm{GeV}$ to $200 \mathrm{eV}$ appears to be pulsed and thus the simplest expectation is that even the $2 \mathrm{eV}$ optical radiation should be pulsed. In accretion-disk models, the optical radiation could arise from the accretion disk and the high-energy radiation closer to the degenerate star. The absence of an optically detectable companion notwithstanding, accretion models have difficulty in explaining the absence of $\mathrm{H} \alpha$ emission (DK) and the very low ratio of optical flux to the total flux (Katz 1985). To summarize, our stringent limits on possible pulses from the $\mathrm{G}$ candidate make it unlikely that it is the optical counterpart to the pulsed x-ray source.

There are several reasons independent from the one advanced here suggesting that the $G$ candidate is probably unrelated to $1 \mathrm{E} 0630+178$ (and hence Geminga as well): (1) absence of an unusual spectrum (Halpern, Grindlay, and Tytler 1985; DK) (2) lack of parallax and proper motion (Sol et al. 1985; DK), (3) lack of unusual colors (Halpern, Grindly, and Tytler 1985; Sol et al. 1985) and (4) lack of variability (Sol et al. 1985 and this work). The colors and the spectroscopy indicate that the $G$ candidate is either a white dwarf or a weak-lined G-type subdwarf (DK). It is fair to say that none of these reasons by themselves can rule out the $G$ candidate as the optical counterpart of $1 E$ $0630+178$, but treated collectively, they strengthen point (b) above. Thus we are left with the interesting result that the optical counterpart of Geminga or of $1 E 0630+178$ at least is either the $24.3 \mathrm{mag}$ star $\mathrm{G}^{\prime}$ (see Table II; also Sol et al. 1985 and DK), or the $>25 \mathrm{mag}$ star $\mathrm{G}^{\prime \prime}$ discussed in the companion paper (DK), or some other star fainter than $\mathrm{G}^{\prime \prime}$. If $G^{\prime}$ is the optical counterpart of the $x$-ray source, then the $\mathrm{x}$-ray to optical flux is about 1100 ; this ratio is considerably higher if the optical counterpart is either $\mathrm{G}^{\prime \prime}$ or some other yet fainter star. An $L_{x} / L_{\text {opt }}$ of $10^{3}$ is characteristic of the low-mass $x$-ray binary systems; however, the absolute visual magnitude of such systems is about 3 (Bradt et al. 1983). Thus, regardless of the periodicity controversy, the source $1 \mathrm{E} 0630+178$ is interesting because, while its $L_{x} / L_{\text {opt }}$ is characteristic of the low-mass $x$-ray binaries, it appears to be about at least 16 mag fainter than the low-mass x-ray binaries.

What could be the nature of the $\mathrm{x}$-ray source? The $\mathrm{x}$-ray and the optical emission may be explained as thermal emission by a neutron star but the $\gamma$-ray flux clearly indicates nonthermal processes (DK; Katz 1985). Katz (1985) has suggested that Geminga is an old spun-down radio pulsar which has now turned off radio emission and is presently converting its rotational energy efficiently into $\gamma$ rays; the $x$ ray and optical emission is assumed to be predominantly nonthermal in nature. This model fits in nicely with the tentative finding by $\mathrm{DK}$ that star $\mathrm{G}^{\prime}$ may have a detectable proper motion. DK argue that the detection of proper motion of $G^{\prime}$, despite its apparent faintness, implies that $G^{\prime}$ is a very compact star like a neutron star. However, there are two difficulties with a single rotating neutron-star model with a period as large as $60 \mathrm{~s}$ :

(a) The rotational energy of a neutron star is $\sim 5.5 \times 10^{42}$ $(P / 60 \mathrm{~s})^{-2} \mathrm{erg} \mathrm{s}^{-1}$. Assuming a distance of $100 \mathrm{pc}$ for $\mathrm{Ge}-$ minga, the observed $\gamma$-ray flux leads to a dynamic age of $\sim 333 \mathrm{yr}$. The age of the x-ray object $1 \mathrm{E} 0630+178$, assuming it is unrelated to Geminga, is a thousand times longer, i.e., $3 \times 10^{5} \mathrm{yr}$. Thus we are either singularly fortunate to be able to observe either the $\mathrm{x}$-ray or the $\gamma$-ray source, or the period of the neutron star is much lower than $60 \mathrm{~s}$.

(b) The maximum potential drop that is available in a rotating, magnetic neutron-star magnetosphere is $1.7 \times 10^{8} B_{12}$ $(P / 60)^{-2} \mathrm{~V}$, where $B_{12}$ is the surface magnetic field strength in units of $10^{12}$ Gauss and $P$ is the period in seconds (Manchester and Taylor 1977). Most of the radio pulsars have $B_{12}>1$ and there appears to be a cutoff around $B_{12}>10$. Thus the minimum magnetic field strength required for the production of a 1 to $3 \mathrm{GeV}$ photon (such as the ones seen by $C O S-B$ towards Geminga) through any process involving acceleration of electrons requires $B_{12}>13$. The true magnetic field strength must surely be many times this minimum field strength since the minimum field-strength calculation assumes efficient acceleration of electrons to the maximum possible energy followed by full conversion of the energy of the electron into a single photon. Alternatively, the $\gamma$ rays are produced by energetic highly charged ions (an unlikely possibility). Thus either Geminga could be a slowly rotating neutron star with a super-strong magnetic field, or the period is much less than $60 \mathrm{~s}$. Thus while Katz's model is attractive for many reasons it runs into a problem if Geminga (and $1 \mathrm{E} 0630+178$ ) does indeed have a rotational period of $60 \mathrm{~s}$. Finally, we would like to point out that if either $\mathrm{G}^{\prime}$ or $\mathrm{G}^{\prime \prime}$ is the correct optical counterpart of $1 \mathrm{E} 0630+178$, then timeresolved photometry of $\mathrm{G}^{\prime}$ or $\mathrm{G}^{\prime \prime}$ should show the same signature as the $x$-ray source. Due to the two-dimensional nature of our data, the data we obtained can, in principle, be used to search for modulated emission from these two stars; however, the faintness of the two stars has denied us this opportunity.

Dan Werthimer was principally responsible for the design and fabrication of the shutter-controller mechanism. We are much indebted to him for this and also gratefully acknowledge him for the loan of his synthesizer and help in observations. $H$. Spinrad made generous contributions of observing time at Lick. We would like to thank C. Heiles for discussion about the data analysis and J. Arons for stimulating conversations on theoretical issues. The "phased"-image observations required many changes at Lick. We are grateful to the staff of Lick for their cooperation and help, and in particular to R. Stone, K. Baker, W. Earthman, J. Morey, C. Clark, and B. Alcott. We thank A. Klemola for his help with astrometry. A major portion of the data reductions were done with VISTA, a software package developed by R. Stover, T. Lauer, and D. Terndrup of the Lick Observatory. This work was supported in part by NSF Grants Nos. AST 82-12058 and AST 84-16863 and a University of California Regents Fellowship to S.D. 


\section{REFERENCES}

Arons, J. (1985). Preprint.

Bignami, G. F., and Hermsen, W. (1983). Annu. Rev. Astron. Astrophys. 21, 67.

Bignami, G. F., Caraveo, P. A., and Lamb, R. C. (1983). Astrophys. J. Lett. 272, L9.

Bignami, G. F., Caraveo, P. A., and Paul, J. A. (1984). Nature 310, 464.

Bisnovaty-Kogan, G. S. (1985). Nature 315, 555.

Bloemen, J. B. G. M. (1984). Astrophys. Astron. 131, L7.

Bradt, H. V. D., and McClintock, J. E. (1983). Annu. Rev. Astron. Astrophys. 21, 13.

Bucheri, R., D'Amico, N. D., Hermsen, W., and Sacco, B. (1985). Nature 316, 131.

Caraveo, P. A. Bignami, G. F., Vigroux, L., and Paul, J. A. (1984). Astrophys. J. Lett. 276, L45.

Djorgovski, S. (1985). Publ. Astron. Soc. Pac. (in press).

Djorgovski, S., and Kulkarni, S. R. (1986). Astron. J. 91, 90.

Halpern, J. P., Grindlay, J. F., and Tytler, D. (1985). Astrophys J. 296, 190.

Katz, I. (1985). Astrophys. Lett. 24, 183.
Kulkarni, S. R., and Djorgovski, S. (1985). IAU Circ. No. 4058.

Manchester, R. N., and Taylor, J. H. (1977). Pulsars (Freeman, San Francisco).

Maraschi, L., and Treves, A. (1977). Astron. Astrophys. 61, L11.

Masnou, J. L., et al. (1977). Proceedings of the 12th ESLAB Symposium, ESA SP-124 (European Space Agency, Noordwijk, The Netherlands), p. 33.

Masnou, J. L., et al. (1981). 17th I. C. R. C. Paris 1, 177.

Miller, J. (1983). Lick Observatory Internal Report.

Nulsen, P. E. J., and Fabian, A. C. (1984). Nature 312, 48.

Peterson, B.A., et al. (1978). Nature 276, 475.

Sol, H., Tarenghi, M., Vanderriest, C., Vigroux, L., and Lelievre, G. (1985). Astrophys. Astron. 144, 109.

Stone, R. P. S. (1977). Astrophys. J. 218, 767.

Thompson, D. J., Fichtel, C. E., Hartman, R. C., Kniffen, D. A., and Lamb, R. C. (1977). Astrophys. J. 213, 252.

Vigroux, L., Paul, J. A., Delache, P., Bignami, G. F., Caraveo, P. A., and Salotti, L. (1984). Preprint. 